\title{
DblurDoseNet: A Deep Residual Learning Network for Voxel Radionuclide Dosimetry Compensating for SPECT Imaging Resolution
}

Zongyu Li ( $\square$ zonyul@umich.edu )

University of Michigan https://orcid.org/0000-0003-1813-1722

Jeffrey A. Fessler

University of Michigan

Justin K. Mikell

University of Michigan

Scott J. Wilderman

University of Michigan

Yuni K. Dewaraja

University of Michigan

\section{Research Article}

Keywords: Deep learning, Voxel-level dosimetry, Lu-177 therapy, SPECT resolution effects

Posted Date: March 17th, 2021

DOI: https://doi.org/10.21203/rs.3.rs-304351/v1

License: (c) (1) This work is licensed under a Creative Commons Attribution 4.0 International License.

Read Full License

Version of Record: A version of this preprint was published at Medical Physics on December 9th, 2021.

See the published version at https://doi.org/10.1002/mp.15397. 
DblurDoseNet: A Deep Residual Learning Network for Voxel Radionuclide Dosimetry Compensating for SPECT Imaging Resolution

Zongyu Li ${ }^{1}$, Jeffrey A. Fessler ${ }^{1}$, Justin K. Mikell ${ }^{2}$, Scott J. Wilderman ${ }^{3}$, Yuni K. Dewaraja ${ }^{4}$

${ }^{1}$ Department of Electrical Engineering and Computer Science, ${ }^{2}$ Radiation Oncology, ${ }^{3}$ Department of Nuclear Engineering and Radiologic Sciences, ${ }^{4}$ Department of Radiology, University of Michigan, Ann Arbor, MI, United States.

Corresponding Author:
Zongyu Li

1301 Catherine, 2276 Medical Science I/5610

Ann Arbor, MI 48109

Email: zonyul@umich.edu

Phone: 734-223-1155

ORCID: 0000-0003-1813-1722 


\section{Abstract}

Purpose: Current methods for patient specific voxel-level dosimetry in radionuclide therapy suffer from a trade-off between accuracy and computational efficiency. Monte Carlo (MC) radiation transport is considered as the gold standard but is computationally expensive, while faster dose voxel kernel (DVK) convolution can be sub-optimal in the presence of tissue heterogeneities. Furthermore, the accuracies of both these methods are limited by the spatial resolution of the reconstructed emission image. To overcome these limitations, this paper takes a novel approach of constructing a single deep convolutional neural network (CNN) named as DblurDoseNet that learns to produce dose-rate maps while compensating for the limited resolution of SPECT images. Methods: To mitigate the effects of poor SPECT resolution and reconstruction artifacts on dosimetry, we trained our CNN using MC-generated dose-rate maps that directly corresponded to the true activity maps in virtual patient phantoms. We applied residual learning such that our CNN only learned the difference between the true dose-rate map and DVK dose-rate map with density scaling. The network consists of a depth feature extractor and a 2D U-Net, where the input was 11 slices $(3.3 \mathrm{~cm})$ of Lu-177 SPECT/CT images and the output was the dose-rate map corresponding to the center slice. In addition to phantoms, 42 SPECT/CT scans of patients who underwent Lu-177 DOTATATE therapy were also used for testing. Results: In test phantoms, the lesion/organ mean dose-rate error and the normalized root mean square error (NRMSE) relative to ground-truth for the CNN method was consistently lower than DVK and MC. In particular, for CNN compared to DVK/MC, the average improvement in mean dose error was $55 \% / 53 \%$ and $66 \% / 56 \%$; and in NRMSE was $18 \% / 17 \%$ and $10 \% / 11 \%$ for lesion and kidney, respectively. Line profiles and dose-volume histograms demonstrated compensation for SPECT resolution effects in the CNN generated dose-rate maps. Noise, determined from multiple Poisson realizations, showed an average improvement of $21 \% / 27 \%$ compared to DVK/MC. In patients, a high concordance was observed between $\mathrm{CNN}$ and $\mathrm{MC}$ in joint histogram analysis. The trained 
residual CNN took $\sim 30$ seconds on GPU to generate a $(512 \times 512 \times 130)$ dose-rate map for a patient. Conclusion: The proposed CNN is well-suited for real-time patient-specific dosimetry for clinical treatment planning due to its demonstrated improvement in accuracy, resolution, noise and speed over the current gold-standard.

Keywords: Deep learning, Voxel-level dosimetry, Lu-177 therapy, SPECT resolution effects 


\section{Introduction}

Accurate and computationally efficient methods for patient specific absorbed dose estimation is paramount for clinical implementation of dosimetry guided treatment planning in radionuclide therapy. For example, current Lu-177 DOTATATE therapy for neuroendocrine tumor is administered on a fixed activity basis ( 4 cycles of $7.4 \mathrm{GBq}$ ), but SPECT/CT imaging-based dosimetry after one cycle can be used to individualize the next administration to potentially enhance tumor response while keeping toxicity to critical organs like kidney at an acceptable level [1]. Traditionally, the mean absorbed dose in volumes of interest (VOIs) is the reported quantity, but voxel-level calculation enables consideration of multiple alternative dose metrics, such as statistics from dose-rate volume histogram (DRVH) analysis that is potentially more relevant for treatment planning. Explicit Monte Carlo (MC) radiation transport using the patient's emission (PET or SPECT) and anatomical images (CT) as input is broadly accepted as the gold standard for voxel-level patient-specific dosimetry, however, it is computationally expensive to generate estimates with low statistical uncertainty. In contrast, faster and simpler dose voxel kernel (DVK) convolution methods [2] can be inaccurate in the presence of heterogeneous tissues, i.e., at the liver-lung or bone-marrow interfaces. Moreover, both these methods, including direct MC that is theoretically accurate, is impacted by reconstruction artifacts and the poor spatial resolution of SPECT and PET, which degrades the dose estimation accuracy.

Over the past few years, deep learning has impacted many fields in medical imaging [3,4]. For example, one popular deep neural network in medical imaging is the U-Net [5] that achieved state-of-the-art accuracy on international symposium on biomedical imaging (ISBI) challenge for segmentation of neuronal structures in electron microscopic stacks. The U-Net has a welldesigned combination of down-sampling and up-sampling layers and can be trained in an end-toend way. Recently, there is an increase of interest in studies that apply deep neural networks in nuclear medicine applications [6-8]. However, deep learning applications [9,11-13] in radionuclide 
therapy are limited. Akhavanallaf et al [9], employed a modified ResNET [10] that represented voxel S-value kernels [2] to predict the distribution of the deposited energy in whole-body organlevel dosimetry and demonstrated comparable performance to the direct MC approach. Lee et al [11], implemented a 3D U-Net [5] that used PET and CT-based density image patches to predict 3D voxel-level dose-rate maps. Götz et al [12], proposed a hybrid method based on a combination of the modified U-Net and empirical mode decomposition of density maps to enhance the accuracy/reliability of radiation dose estimation. Despite promising results, a potential limitation of the training approaches in these prior studies $[11,12]$ is that they used MC-generated doserate maps derived from patient's measured SPECT or PET images as the training label, which are degraded by poor spatial resolution and reconstruction artifacts. Similarly, the dose-rate map in [9] was derived by convolving the CNN-based S-value kernels with patient's PET activity map, so it also suffers from issues mentioned above. In contrast, by using dose-rate maps that directly correspond to the true activity maps as the ground-truth in training, a CNN dosimetry model has the potential to overcome limitations associated with SPECT or PET spatial resolution and reconstruction artifacts.

The aim of this study was to develop a deep learning-based absorbed dose-rate estimation method that can overcome the accuracy-efficiency trade-off associated with current voxel dosimetry methods. At the same time, our proposed CNN, DblurDoseNet, also attempts to learn to reduce the degrading effects of spatial resolution and reconstruction artifacts by using dose-rate estimates directly corresponding to phantom (virtual patient) activity maps as the training label, instead of the patient SPECT-derived dose-rate images (Fig. 1). Furthermore, unlike prior dosimetry studies where CNN was trained to directly estimate the dose-rate map or S-value kernels, we first used the physics-based fast Fourier transform (FFT) DVK convolution method (with density scaling) to produce initial estimates, and then trained the CNN to learn the subtle residual differences between the initial estimate and the true dose-rate maps. We trained 
and tested the proposed CNN for SPECT/CT imaging-based dosimetry following Lu-177 DOTATATE therapy of neuroendocrine tumors (NETs).

\section{Material and methods}

\section{Virtual patient phantom generation for training and testing}

Fig. 2 is an overview of our data generation and training process. We chose to use PET instead of SPECT-based activity maps to generate the virtual patient phantoms because PET offers substantially higher spatial resolution than SPECT as evident in top branch of Fig. 2 . These images were readily available because prior to Lu-177 DOTATATE, patients underwent diagnostic Ga-68 DOTATATE PET/CT imaging (Siemens Biograph mCT) to determine eligibility for therapy. The Ga-68 DOTATATE distribution in patients is expected to be similar to the Lu177 DOTATATE distribution. Thus, we selected 14 such PET scans from our clinic database to generate phantoms for training and testing, with University of Michigan Institutional Review Board (IRB) approval for retrospective analysis. The selected cases covered a diverse range with regards to sex, size and lesion location (within and outside liver). The PET/CT images were then extracted into 130 slices that covered the SPECT field-of-view $(39 \mathrm{~cm})$ with liver and kidney centered, which is the typical region imaged following Lu-177 DOTATATE. Meanwhile, the corresponding density maps were generated using an experimentally derived CT-to-density calibration curve.

Next, Lu-177 SPECT projections corresponding to phantom's activity/density maps were generated using the SIMIND MC code [14] (Fig. 2 top branch) simulating approximately 2 billion histories per projection. Parameters used for Lu-177 patient imaging in our clinic (Siemens Intevo with medium energy collimators, a 5/8" crystal, a 20\% photopeak window at $208 \mathrm{keV}$ and two adjacent $10 \%$ scatter windows) were modeled. Poisson noise was added after the 120 projection 
views were scaled to a count-level in the range of 3 to 20 million total counts, which corresponds to the range in post-therapy imaging. SPECT reconstruction used an in-house 3D OSEM algorithm with CT-based attenuation correction, triple energy window scatter correction and collimator-detector response modeling (4 subsets and 16 iterations, $128 \times 128 \times 81$ matrix with voxel size $4.8 \times 4.8 \times 4.8 \mathrm{~mm}^{3}$, no gaussian smoothing). All images were finally registered into CT image space $\left(512 \times 512 \times 130\right.$ with voxel size $\left.0.98 \times 0.98 \times 3 \mathrm{~mm}^{3}\right)$.

Out of 14 virtual patient phantoms, 9 were used for training and 5 for testing. Out of the training dataset, to assess under/over-fitting, we randomly selected $20 \%$ of total slices to serve as validation dataset.

\section{Patient data}

In addition to the above virtual patients, a total of 42 scans from 12 patients imaged at up to 4 time points during the first week following cycle 1 of standard Lu-177 DOTATATE (7.4 GBq) were included in our testing dataset. The images were acquired as part of an ongoing University of Michigan IRB approved research study, where all subjects signed an informed consent form. SPECT acquisition time was $25 \mathrm{~min}$ and all other imaging/reconstruction parameters were as described above for the phantom simulation. The CT was performed in low-dose mode (120 kVp; $15-80 \mathrm{mAs}$ ) with free breathing.

\section{Monte Carlo dosimetry and dose voxel kernel convolution}

Monte Carlo. The Monte Carlo code that we used, Dose Planning Method (DPM), was originally developed and validated for fast dose-rate estimation in external beam radiotherapy [15]. Previously, we adapted and benchmarked DPM for the internal radionuclide therapy application [16]. Because DPM was optimized specifically for voxel-level electron/photon dose computations with full radiation transport, it is faster than using general-purpose $\mathrm{MC}$ codes for voxel-level dose 
estimation. We used DPM to generate the ground truth training labels (Fig. 2) simulating 1 billion histories to generate dose-rate maps with low statistical uncertainty.

DVK convolution with density scaling. To provide DVK dose-rate maps for residual learning, Lu177 soft tissue $\left(1.04 \mathrm{~g} / \mathrm{cm}^{3}\right)$ voxel kernels were generated by the DPM code. The beta particle kernel size was $9 \times 9 \times 9$ and the photon kernel size was $99 \times 99 \times 99$ (both with voxel size $\left.0.98 \times 0.98 \times 3 \mathrm{~mm}^{3}\right)$. We convolved the SPECT image with the DVKs using fast (FFT)convolution. Since using homogeneous soft tissue kernels neglects tissue inhomogeneities, we applied density scaling that has been shown to be a reasonable correction in past reports [17]. Here, after convolution, each voxel was scaled by $1.04\left(\mathrm{~g} / \mathrm{cm}^{3}\right)$ and divided by local voxel density value $\left(\mathrm{g} / \mathrm{cm}^{3}\right)$ derived from $\mathrm{CT}$. Because our goal was to generate a reasonably accurate and quick initial estimate for the residual learning process, other more sophisticated approaches $[18,19]$ that account for tissue heterogeneities were not pursued.

To address the very high dose-rate estimate in extra low-density regions, i.e., air gap, we set the dose-rate in regions of which the density is less than $0.1 \mathrm{~g} / \mathrm{cm}^{3}$ to zero.

\section{Network : DblureDoseNet}

We considered the decay properties of Lu-177 and physics of beta/photon interaction in tissue when designing our network. The mean energy of the emitted electrons in the beta decay of Lu177 is $134 \mathrm{keV}$ and the maximum energy is $497 \mathrm{keV}$, and the corresponding continuous slowing down approximation (CSDA) range (in water) is only $0.3 \mathrm{~mm}$ and $1.8 \mathrm{~mm}$, respectively [20]. The gamma-rays associated with Lu-177 are low in intensity (113 keV (6.2\%) and $208 \mathrm{keV}(10.4 \%)$ ), hence, the absorbed dose is dominated by the beta component.

Furthermore, considering the trade-off between the computational efficiency of CNN and contribution of photons that travel far in tissue, we resized the input SPECT \& density images into 
packs containing 5 adjacent slices for each side with paddings that replicate the first and last slices at the top and bottom boundaries, respectively. Thus, there were two input arrays of size $512 \times 512 \times 11$ (with voxel size $0.98 \times 0.98 \times 3 \mathrm{~mm}^{3}$ ) and one output array of size $512 \times 512$ that corresponded to the dosimetry of the middle slice in the input arrays.

As shown in Fig. 2, we first concatenated the input activity/density maps along the channel dimension, then applied three 3D convolutional layers (with kernel size $7 \times 7 \times 5,7 \times 7 \times 3,7 \times$ $7 \times 3$, respectively) to extract the depth features. Next, we implemented a 2D U-Net that had 4 down-sample and up-sample layers. The first convolutional layer in the 2D U-Net has 16 filters. After each down-sample layer, the number of filters at the next convolutional layer is increased by a factor of 2 until it reaches 128 . Next, we added the DVK dose-rate map to the $2 \mathrm{D} U$-Net output, as the operation of residual learning. Finally, by setting the dose-rate in extra low-density area $\left(\rho<0.1 \mathrm{~g} / \mathrm{cm}^{3}\right)$ to zero, we obtain the CNN dose-rate map estimate.

The CNN was trained by minimizing the mean square error with batch size 32 between the ground-truth and CNN dose-rate map. We applied ADAM optimizer [21] with dynamic learning rate (an initial value 0.001 with ReduceOnPlateau management strategy) and trained our CNN for 200 epochs on two Nvidia Tesla V100 GPUs. The training/validation loss decreased to 288/410 after 4 hours of training (see Supplemental Data Fig. 1). To cover different input count levels, we first normalized every SPECT activity map so that all voxels in each activity map sum to one. To potentially avoid zigzagging during training, we also scaled the normalized SPECT and dose-rate maps with a constant value so that they have a similar range as the density maps. 


\section{Evaluation metrics}

In test phantoms, the dose-rate maps generated by DVK, MC and CNN were evaluated qualitatively by visual comparison of images, line profiles and dose-rate-volume histograms (DRVHs) with those corresponding to the ground truth. For quantitative evaluations, we used the following metrics:

Dose-rate error. For each VOI, the absolute error in the mean dose-rate and DRVH statistics D30, D70 (minimum dose-rate to $30 \%(70 \%)$ of the VOI) were calculated relative to the ground-truth.

Normalized root mean square error (NRMSE).

$$
\text { NRMSE }=\frac{\sqrt{\frac{1}{n_{p}} \sum_{j=1}^{n_{p}}\left(\hat{x}_{j}-x_{j}\right)^{2}}}{\sqrt{\frac{1}{n_{p}} \sum_{j=1}^{n_{p}} x_{j}^{2}}}
$$

Where $n_{p}$ is the total number of voxels in the VOI. Subscript $j$, i.e., $x_{j}$, denotes the $j$ th voxel in the image. The true and the estimated dose-rate image are denoted by $x$ and $\hat{x}$, respectively.

Ensemble noise. The ensemble noise in spherical VOls defined in non-tumoral liver or spleen was calculated across $3(M=3)$ Poisson noise realizations as:

$$
\text { Noise }=\frac{\sqrt{\frac{1}{J_{\mathrm{VOI}}} \sum_{j \in \mathrm{VOI}}\left(\frac{1}{M-1} \sum_{m=1}^{M}\left(\hat{x}_{m}[j]-\frac{1}{M} \sum_{m^{\prime}=1}^{M} \hat{x}_{m \prime}[j]\right)^{2}\right)}}{\frac{1}{J_{\mathrm{VOI}}} \sum_{j \in \mathrm{VOI}} \frac{1}{M} \sum_{m=1}^{M} \hat{x}_{m}[j]} \times 100 \%
$$

Where $\hat{x}_{m}[j]$ denotes the $j$-th voxel in the estimated dose-rate image of the $m$-th Poisson noise realization and $J_{\mathrm{VOI}}$ denotes the total number of voxels in the VOI.

The lesion VOls for these quantitative evaluations were defined manually on CT of SPECT/CT guided by baseline diagnostic CT or MRI by a radiologist with abdomen imaging 
expertise. Organs were defined using semi-automatic CT segmentation tools. The healthy liver was defined as liver minus lesions in the liver.

In patients, where there is no known ground-truth, results were compared visually. In addition, a joint histogram analysis was performed by plotting the intensity of voxels in the CNN dose-rate images versus the corresponding voxels in the $\mathrm{MC}$ estimates. For the joint histogram we report Pearson's correlations and Lin's concordance correlations coefficient (CCC).

\section{Results}

\section{Virtual patient phantom test results}

Qualitative Assessment. Generally, there was better visual agreement between CNN dose-rate maps and the ground-truth than the agreement between DVK/MC dose-rate maps and the ground-truth. Qualitative evidence of the superior performance of the CNN is shown by the example images and line profiles of Fig. 3 and DRVHs of Fig 4.

Quantitative Assessment. The mean dose-rate values for organs/lesions across 5 test phantoms are presented in supplemental Table 1. Fig. 5A compares the absolute mean dose-rate error in lesions and organs across all test phantoms. As evident in qualitative assessment, the CNN also consistently show superior results than DVK and MC. For instance, compared to DVK/MC, CNN estimates show an average improvement of $52 \% / 20 \%, 55 \% / 53 \%, 66 \% / 50 \%, 66 \% / 62 \%, 48 \% / 49 \%$ and $58 \% / 39 \%$ in healthy liver, lesion, left kidney, right kidney, spleen and lumbar, respectively. The absolute error in DRVH statistics (D30 and D70) also demonstrates superior results for the CNN estimate (Supplemental Fig. 2). The NRMSE was also substantially lower for CNN than for DVK and MC across all VOls (Fig. 5B). The average improvement demonstrated by CNN compared to DVK/MC was $10 \% / 9 \%, 18 \% / 17 \%, 11 \% / 12 \%, 9 \% / 10 \%, 26 \% / 27 \%$ and $18 \% / 10 \%$ in healthy liver, lesion, left kidney, right kidney, spleen and lumbar, respectively. In addition to the 
improvement in the average values, the maximum errors (denoted by the error bars in Fig. 5) were also consistently lower with CNN compared to DVK and MC. In Fig. 5 and supplemental Fig. 2, all three methods show the highest errors for lesion and lumbar vertebrae regions. This is attributed to the smaller size of these VOls compared to other organs and the corresponding increase in partial volume effects. In the case of lumbar, relevant to bone marrow dosimetry, the very low uptake in these regions also contributes to higher dose-rate errors.

Noise Evaluation. Table 1 shows a consistent reduction of ensemble noise in background VOls with an average of $21 \%$ and $27 \%$ improvement demonstrated by CNN compared to DVK and MC, where MC had the highest level of noise due to its statistical nature.

Table 1. Ensemble noise from 3 realizations for DVK, MC and CNN across all test phantoms. Number of voxels ranges from 2527 to 23411.

\begin{tabular}{|c|c|c|c|c|}
\hline Ensemble Noise & $\begin{array}{c}\text { Background } \\
\text { Region }\end{array}$ & DVK & MC & CNN \\
\hline Phantom \#1 & Liver \& Spleen & $4.6 \%$ & $6.1 \%$ & $\mathbf{3 . 4 \%}$ \\
\hline Phantom \#2 & Liver & $12.6 \%$ & $13.3 \%$ & $\mathbf{9 . 2} \%$ \\
\hline Phantom \#3 & Liver & $14.0 \%$ & $14.6 \%$ & $\mathbf{1 2 . 9 \%}$ \\
\hline Phantom \#4 & Liver & $20.3 \%$ & $19.6 \%$ & $\mathbf{1 4 . 8 \%}$ \\
\hline Phantom \#5 & Spleen & $7.1 \%$ & $7.6 \%$ & $\mathbf{5 . 8 \%}$ \\
\hline
\end{tabular}

\section{Patient test results}

Fig. 6 shows examples of dose-rate maps corresponding to high count (day 1 post-therapy) and low-count (day 7 post-therapy) imaging conditions post-Lu-177 DOTATATE. Although concrete 
conclusions cannot be drawn as there is no known truth, visual inspections imply potential reduction of SPECT spatial resolution effects on dose-rate accuracy by our DblurDoseNet. For instance, with the CNN, the enlarged kidney map and line profiles of Fig. 6A show a larger decrease in dose-rate in the medulla and renal pelvis areas, which can be due to the expected lower physiological Lu-177 uptake in this part of the kidney compared to the cortex region. In addition, in Fig. 6B, the lesion with a necrotic center demonstrates a larger drop in dose-rate at the center with the CNN compared to DVK or MC, which can be due to the expected lower uptake associated with necrosis.

The dose-rate values for all patient VOls are presented in supplemental Table 2. Fig. 7 shows the joint histogram between $\mathrm{CNN}$ and $\mathrm{MC}$ dose-rate maps across all voxels in evaluated VOIs for all test patients and demonstrates a very strong agreement (Pearson $R^{2}=0.95$, Lin's Concordance Correlation Coefficient $(C C C)=0.97)$.

\section{Comparing performance with residual vs. non-residual training}

We examined the effectiveness of residual learning framework by comparing the previous test phantom results to those generated with a CNN with the same architecture but without residual learning (not adding the DVK dose-rate map to the output of 2D U-Net). The non-residual CNN was trained using the same hyper-parameters and took the same SPECT and density map as input as the residual CNN did. As shown in supplemental Fig. 1, after 200 epochs of training, the training/validation loss of non-residual CNN went down to 902/1250 at the last 50 epochs, compared to $288 / 410$ with residual learning. Besides, as shown in Table 2, quantitative comparisons across all test phantoms shows superior results with residual learning for all VOls except for lumbar where both networks showed similar errors. 
Table 2. Absolute mean dose-rate error and NRMSE comparison between CNN with and without residual learning evaluated across VOIs in all test phantoms, with maximum error showed in the parenthesis.

\begin{tabular}{|c|c|c|c|c|}
\hline Evaluation & \multicolumn{2}{|c|}{ Mean Dose-rate Error } & \multicolumn{2}{|c|}{ NRMSE } \\
\hline Organ & CNN w/ res & CNN w/o res & CNN w/ res & CNN w/o res \\
\hline Healthy Liver & $1.4 \%(2.3 \%)$ & $5.5 \%(7.0 \%)$ & $19.6 \%(33.2 \%)$ & $21.6 \%(35.1 \%)$ \\
\hline Lesion & $5.3 \%(13.0 \%)$ & $6.9 \%(12.5 \%)$ & $21.2 \%(32.5 \%)$ & $21.4 \%(31.5 \%)$ \\
\hline Liver & $1.9 \%(3.5 \%)$ & $5.7 \%(7.6 \%)$ & $20.6 \%(26.3 \%)$ & $21.6 \%(27.6 \%)$ \\
\hline Left Kidney & $0.9 \%(2.1 \%)$ & $5.2 \%(6.5 \%)$ & $19.2 \%(22.9 \%)$ & $20.1 \%(22.0 \%)$ \\
\hline Right Kidney & $1.8 \%(5.1 \%)$ & $5.8 \%(12.6 \%)$ & $19.6 \%(21.5 \%)$ & $20.5 \%(24.3 \%)$ \\
\hline Spleen & $2.5 \%(6.2 \%)$ & $6.3 \%(9.5 \%)$ & $13.1 \%(17.7 \%)$ & $14.4 \%(19.9 \%)$ \\
\hline Lumbar & $11.1 \%(27.4 \%)$ & $10.5 \%(27.2 \%)$ & $33.0 \%(51.4 \%)$ & $32.9 \%(49.1 \%)$ \\
\hline
\end{tabular}

\section{Time cost}

We compared the time cost among different methods by computing a dose-rate map corresponding to the typical $512 \times 512 \times 130$ patient SPECT/CT image size on CPU (Intel Core i9 @2.3 GHz) or GPU (Tesla V100 GPU): DVK with density scaling took 20 seconds on CPU and 10 seconds on GPU. DPM MC code took $\sim 60$ minutes simulating 1 billion histories on CPU while running DPM on GPU is not an option at this time (we are unaware of any MC code for internal therapy running on GPU). The CNN took 20 minutes on CPU and $\sim 20$ seconds using a GPU. If considering the DVK pre-computation time for the residual learning network, the total GPU time cost for CNN with residual learning is $\sim 20+10$ seconds. 


\section{Discussion}

With evaluation both on virtual patient phantoms that covered clinically relevant conditions and patients who underwent Lu-177 DOTATATE therapy in our clinic, we demonstrated that our CNN using residual learning framework can be applied for fast and accurate dose-rate estimation. Despite using only moderate amount of training data, DbureDoseNet provided consistently superior performance over conventional voxel dosimetry in terms of resolution, accuracy and noise. Importantly, for clinical implementation, the CNN voxel dose-rate map for a $512 \times 512 \times$ 130 patient image could be quickly generated in $\sim 30$ seconds, which is a fraction of the time associated with running $\mathrm{MC}$, the current gold standard. Although generating the ground-truth labels for training by $\mathrm{MC}$ is computationally expensive, this is needed only once at training time, for a given SPECT imaging system.

The main limitation to accurate voxel-level patient specific dose-rate estimation with nonlearning based methods is the poor spatial resolution associated with the input SPECT (or PET) images. This issue was evident in our results where the theoretically accurate MC-based calculation only slightly outperformed DVK with density scaling (Fig. 5 and supplemental Fig. 2). In contrast, by using the true activity map-based dose-rate estimates in training, our CNN has the ability to "learn" the physics of dose deposition and the compensation for the SPECT resolution effects that both lead to blurring of the dose-rate maps, as demonstrated in the test phantom results (Fig. 3-5). In patient studies, potential mitigation of SPECT resolution effects was demonstrated empirically. In Fig. 6, the CNN-based estimates showed sharper line profiles and larger drops in dose-rate over the medulla area of the kidney, analogous to the illustration of Fig 1 , and the necrotic center of a tumor, which is consistent with what is expected based on physiology. The generalizability of the CNN was demonstrated by the high concordance between $\mathrm{MC}$ and $\mathrm{CNN}(\mathrm{CCC}=0.97)$ in joint histogram analysis across $42 \mathrm{SPECT} / \mathrm{CT}$ scans from patients with diverse anatomy/physiology and imaging conditions (day 0 to 7 post-therapy). Although test 
results were promising over 42 scans originating from 12 patients, further testing is planned as more patient images become available.

The mean dose-rate error shown in Fig. 5, especially for lesions, are generally lower than one would expect based on reported recovery coefficients in quantitative Lu-177 SPECT phantom studies. For example, for 72 OSEM updates, activity recovery of only $80 \%$ was reported for a 26.5 $\mathrm{mL}$ volume "hot" sphere in a "warm" background region [22]. The results of the current study show lower errors because, unlike in a physical phantom, the assigned "true" activity at the boundary of the structures in the virtual patients do not drop off sharply, instead, are blurred out. Moreover, in Fig. 5, all 3 methods show the largest mean dose-rate error for lesions and lumbar, as expected due to the relatively small size of these structures, hence larger the impact of spatial resolution. The large error for lumbar with VDK ( 25\%) is probably because of the heterogenous tissue within this region that includes cortical bone, trabecula bone, yellow and red marrow. With VDK, the simple density scaling that was performed in our study is potentially inadequate for this region. Furthermore, the Lu-177 uptake in lumbar region is very low, hence, the cross-dose contribution to dose-rate in lumbar, including the photon cross-dose, can be significant. In this case, it is possible that our $99 \times 99 \times 99$ photon kernel was insufficient to capture the full photon cross dose contribution to the lumbar, which is another possible reason for the relatively large dose-rate error for the lumbar region.

To define our virtual patient activity maps, we chose to use Ga-68 DOTATATE PET/CT to exploit the availability of these images that have higher resolution than SPECT and are expected to show similar uptake patterns as Lu-177 DOTATATE. Ideally, however, images of higher resolution than clinical PET should be used as the true representation of the activity map of patients when generating the virtual patient training set, but this is not readily available. To circumvent this issue, we also investigated using phantoms with piece-wise uniform uptake in CTdefined organs/lesions for training (such as XCAT [25] in Fig. 1), but we found that such training 
leads to very uniform dose-rate maps when tested on patient images. A possible alternative to our PET-based virtual patient activity maps is to assign distributions based on high resolution animal models, for instance, ex-vivo autoradiography showing uptake distribution of DOTATATE in kidney [23].

Our results also demonstrated the advantage of residual learning framework exploiting the fast DVK approach as an initial estimate, which was not utilized in the prior studies [9,11-13]. An alternative to fast DVK for the initial estimate is to generate a quick MC (low number of histories) estimate, which was not explored here. Another advantage of our network is that we first implemented a couple of depth feature extractor layers that shrink the 3D input into $2 \mathrm{D}$ at the beginning of our network. Compared to fully 3D approaches, this approach leads to a network having fewer parameters (because 2D kernels have fewer parameters than $3 D$ kernels), so it is less likely to overfit the training data, avoiding a common problem in deep learning applications for medical imaging, where only moderate amount of training data is available. Alternatively, if further investigating such designs, one direction could be 2.5D CNN architectures [24].

We expect that training a single CNN, as we did in the current study, is simpler than training 2 separate CNNs to learn the dosimetry and SPECT resolution effects. Because typically there would be 3 stages needed to train 2 separate CNNs; Stage 1: training CNN-A for SPECT resolution; stage 2: training $\mathrm{CNN}-\mathrm{B}$ for dosimetry; stage 3: jointly fine-tuning CNN-A and CNN-B. Compared to our proposed end-to-end network, DblurDoseNet, which only involves one training stage, such 3-stage of training would be more complex and potentially inefficient. However, only through comprehensive comparisons can one draws definite conclusions between these two approaches, which is a limitation of our study and we expect to undertake such an evaluation in the future. Although our study only investigated Lu-177 dosimetry, we expect that by changing the training dataset and minor modifications to the architecture, our CNN approach can be 
extended to other radionuclides including $\mathrm{Y}-90$ that is a pure-beta emitters and $\mathrm{I}-131$ that has significant beta and gamma contributions to the dose-rate.

\section{Conclusion}

We constructed and tested a residual CNN that was trained on virtual patient phantom images to learn the mapping from SPECT/CT images to the corresponding dose-rate maps. We took the

novel approach of using a single CNN to learn not only the dose-rate estimation but also to compensate for blurring of the dose-rate map due to poor SPECT resolution. The proposed residual DburDoseNet was able to outperform conventional voxel-level dosimetry methods, including the current "gold standard" MC, in terms of accuracy, noise and speed. Patient specific voxel-level dose rate maps were generated in 30 secs on GPU, hence the CNN approach has much promise for real-time clinical use in radionuclide therapy dosimetry for treatment planning.

Funding information This work was supported by R01 EB022075 awarded by the National Institute of Biomedical Imaging and Bioengineering (NIBIB) and R01 CA240706 awarded by the National Cancer Institute (NCl), $\mathrm{NIH}$.

Data availability The training/testing virtual patient phantoms images will be available at: https://github.com/ZongyuLi-umich/. The CNN was implemented in PyTorch and will be available at: https://github.com/ZongyuLi-umich/. The Image reconstruction algorithm (OSEM) was implemented in MATLAB and is available at https://web.eecs.umich.edu/ fessler/code/index.html. 


\section{Compliance with ethical standards}

Conflict of interest The authors declare that they have no conflict of interest.

Ethical approval All procedures performed in studies involving human participants were in accordance with the ethical standards of the institutional and/or national research committee and with the 1964 Helsinki declaration and its later amendments or comparable ethical standards.

Informed consent Informed consent was obtained from all individual participants included in the study according to University of Michigan Institutional Review Board (IRB) criteria. The current study only involved retrospective analysis of existing SPECT/CT data.

\section{References}

1. Eberlein U, Cremonesi M, Lassmann M. Individualized Dosimetry for Theranostics: Necessary, Nice to Have, or Counterproductive?. J Nucl Med. 2017;58(Suppl 2):97S$103 S$.

2. Bolch WE, Bouchet LG, Robertson JS, et al. MIRD pamphlet No. 17: the dosimetry of nonuniform activity distributions--radionuclide $S$ values at the voxel level. Medical Internal Radiation Dose Committee. J Nucl Med. 1999;40(1):11S-36S.

3. Kortesniemi M, Tsapaki V, Trianni A, et al. The European Federation of Organisations for Medical Physics (EFOMP) White Paper: Big data and deep learning in medical imaging and in relation to medical physics profession. Phys Med. 2018;56:90-93.

4. Litjens G, Kooi T, Bejnordi BE, et al. A survey on deep learning in medical image analysis. Med Image Anal. 2017;42:60-88.

5. Ronneberger O, Fischer P, Brox T. U-net: Convolutional networks for biomedical image segmentation. In MICCAI. 2015; volume 9351 of LNCS: 234-241. 
6. Zukotynski K, Gaudet V, Uribe CF, et al. Machine Learning in Nuclear Medicine: Part 2Neural Networks and Clinical Aspects. J Nucl Med. 2021;62(1):22-29.

7. Veit-Haibach P, Buvat I, Herrmann K. EJNMMI supplement: bringing Al and radiomics to nuclear medicine. Eur J Nucl Med Mol Imaging. 2019;46(13):2627-2629.

8. Arabi, H., Zaidi, H. Applications of artificial intelligence and deep learning in molecular imaging and radiotherapy. European J Hybrid Imaging 4, 17 (2020).

9. Akhavanallaf A, Shiri I, Arabi H, Zaidi H. Whole-body voxel-based internal dosimetry using deep learning. Eur J Nucl Med Mol Imaging. 2020;10.1007/s00259-020-05013-4.

10. He K, Zhang X, Ren S, Sun J. Deep Residual Learning for Image Recognition. In CVPR. 2016; pp. 770-78.

11. Lee MS, Hwang D, Kim JH, Lee JS. Deep-dose: a voxel dose estimation method using deep convolutional neural network for personalized internal dosimetry. Sci Rep. 2019;9(1):10308.

12. Götz TI, Schmidkonz C, Chen S, Al-Baddai S, Kuwert T, Lang EW. A deep learning approach to radiation dose estimation. Phys Med Biol. 2020;65(3):035007.

13. Theresa I. Götz, Elmar W. Lang, Christian Schmidkonz, Torsten Kuwert, Bernd Ludwig. Dose voxel kernel prediction with neural networks for radiation dose estimation. Journal of Medical Physics. Volume 31, Issue 1, 2021, Pages 23-26, ISSN 0939-3889.

14. Ljungberg, M. The SIMIND Monte Carlo Program. 2012. 111-128. 10.1201/b13073-8.

15. Sempau J, Wilderman SJ, Bielajew AF. DPM, a fast, accurate Monte Carlo code optimized for photon and electron radiotherapy treatment planning dose calculations. Phys Med Biol. 2000;45(8):2263-2291 .

16. Wilderman SJ, Dewaraja YK. Method for Fast CT/SPECT-Based 3D Monte Carlo Absorbed Dose Computations in Internal Emitter Therapy. IEEE Trans Nucl Sci. 2007;54(1):146-151. 
17. Dieudonné A, Hobbs RF, Lebtahi R, et al. Study of the impact of tissue density heterogeneities on 3-dimensional abdominal dosimetry: comparison between dose kernel convolution and direct Monte Carlo methods. J Nucl Med. 2013;54(2):236-243.

18. Götz T, Schmidkonz C, Lang EW, Maier A, Kuwert T, Ritt P. A comparison of methods for adapting dose-voxel-kernels to tissue inhomogeneities. Phys Med Biol. 2019;64(24):245011.

19. Lee MS, Kim JH, Paeng JC, et al. Whole-Body Voxel-Based Personalized Dosimetry: The Multiple Voxel S-Value Approach for Heterogeneous Media with Nonuniform Activity Distributions. J Nucl Med. 2018;59(7):1133-1139.

20. Berger BJ, Inokuti M, Anderson HH, et al. Stopping Power for Eletcrons and Positrons, Report 37. Journal of the ICRU. Volume os19, Issue 2, 1 December 1984, Page NP.

21. Kingma DP, Ba J. Adam: A Method for Stochastic Optimization. In ICLR. 2015.

22. Tran-Gia J, Lassmann M. Characterization of Noise and Resolution for Quantitative ${ }^{177} \mathrm{Lu}$ SPECT/CT with xSPECT Quant. J Nucl Med. 2019 Jan;60(1):50-59.

23. Melis M, Krenning EP, Bernard BF, Barone R, Visser TJ, de Jong M. Localisation and mechanism of renal retention of radiolabelled somatostatin analogues. Eur $\mathrm{J}$ Nucl Med Mol Imaging. 2005;32(10):1136-1143.

24. Ziabari A, Ye DH, Srivastava S, et al. 2.5D Deep Learning For CT Image Reconstruction Using A Multi-GPU Implementation. In ACSSC. 2018, pp. 2044-2049.

25. Segars WP, Sturgeon G, Mendonca S, Grimes J, Tsui BM. 4D XCAT phantom for multimodality imaging research. Med Phys. 2010 Sep;37(9):4902-15. 


\section{Figures}

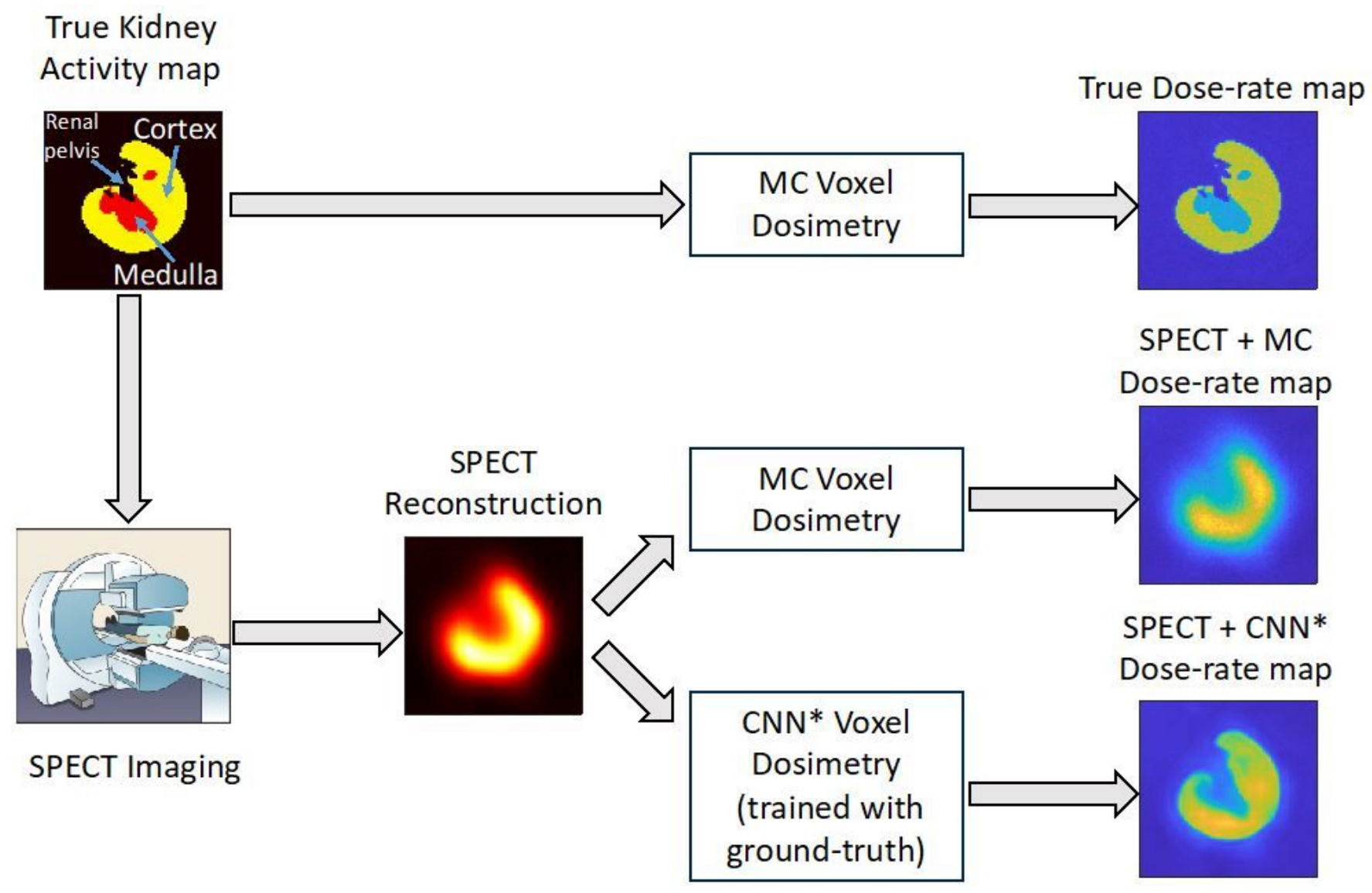

Figure 1

Illustration of blurring of dose-rate maps due to the limited resolution of the SPECT-based input activity map and the potential for a learning-based method to outperform MC, the current gold-standard. CNN* was trained and tested on different XCAT [25] phantoms. 


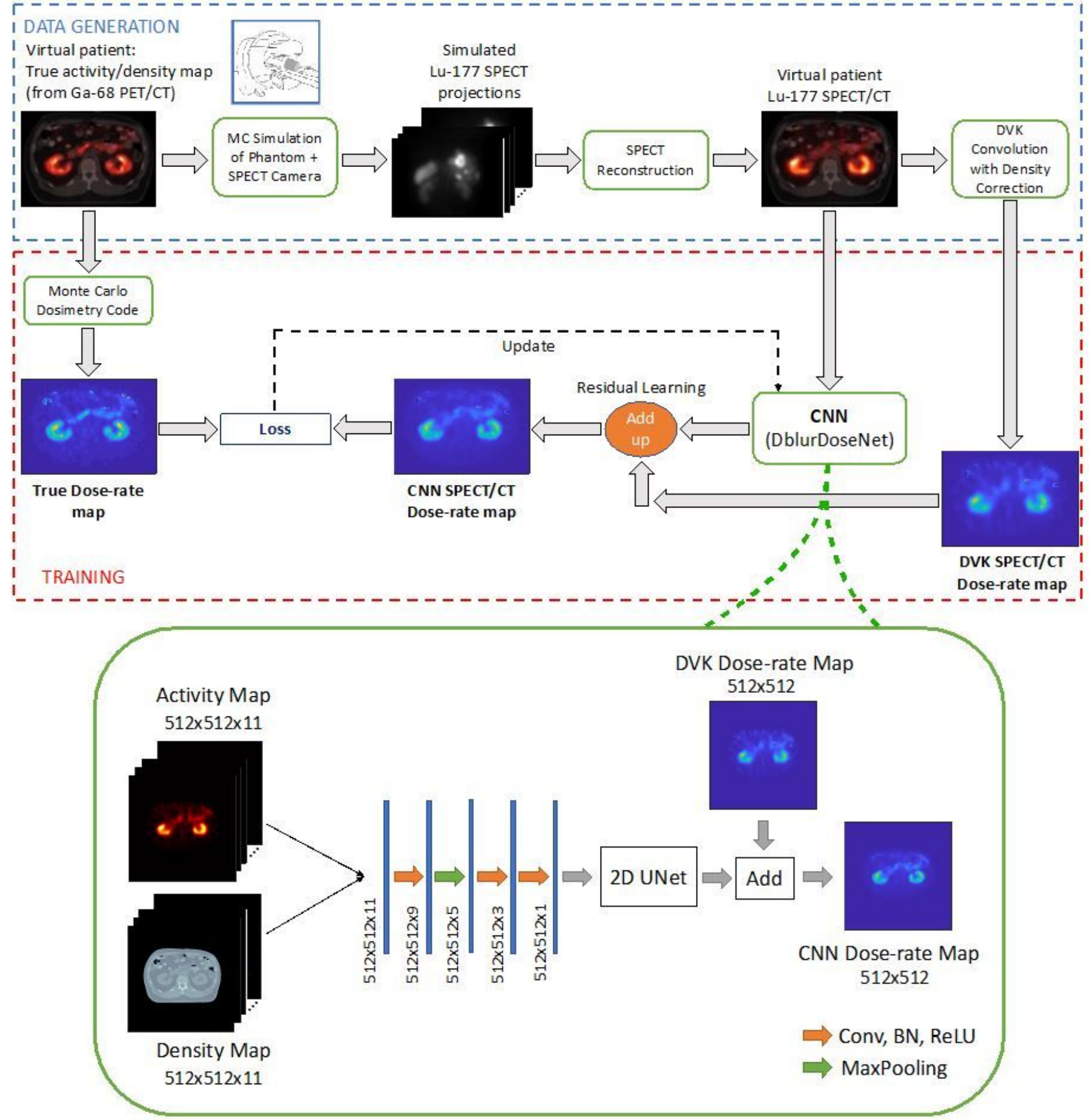

Figure 2

Overview of phantom data generation for training/testing and the network training process. 

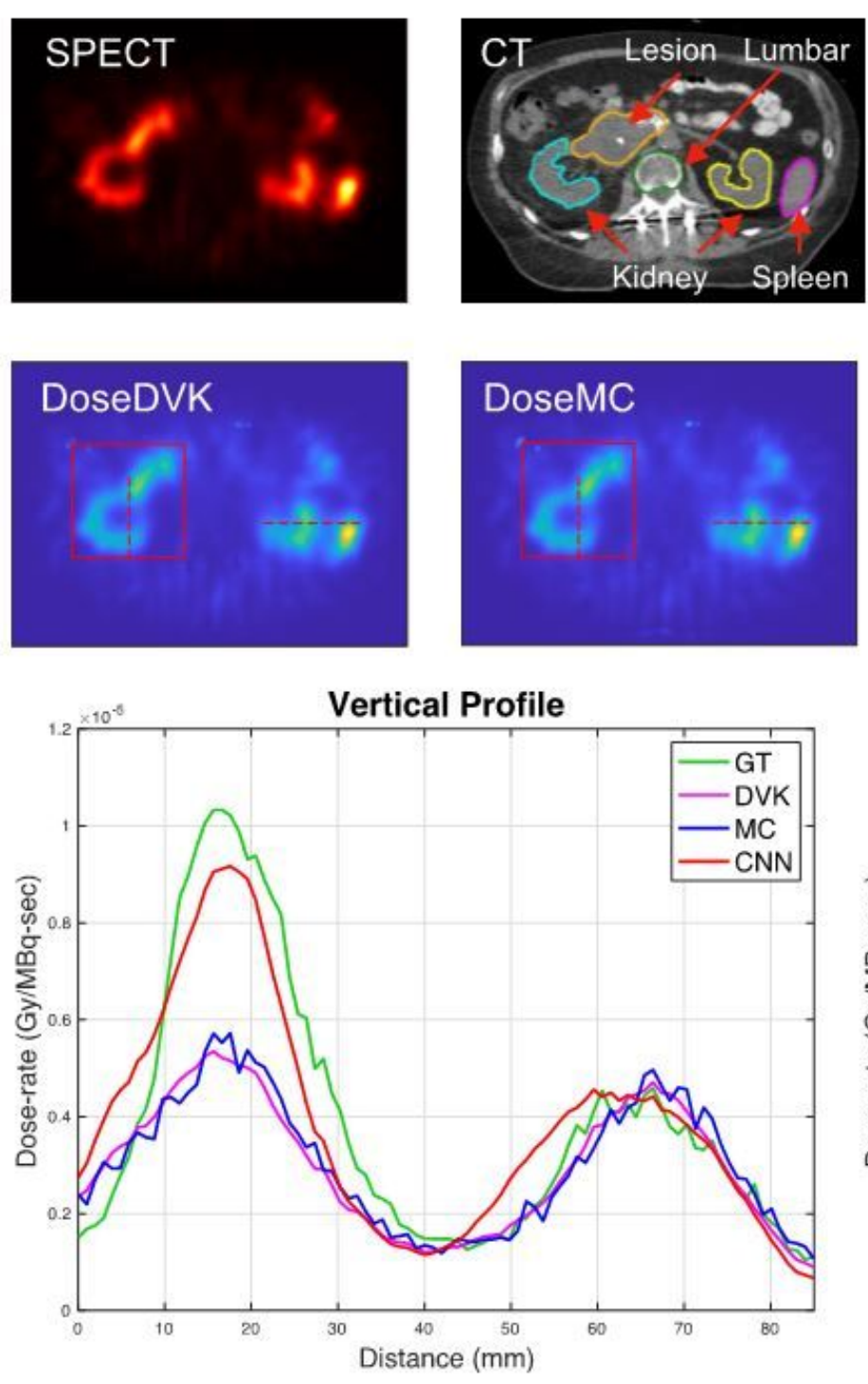
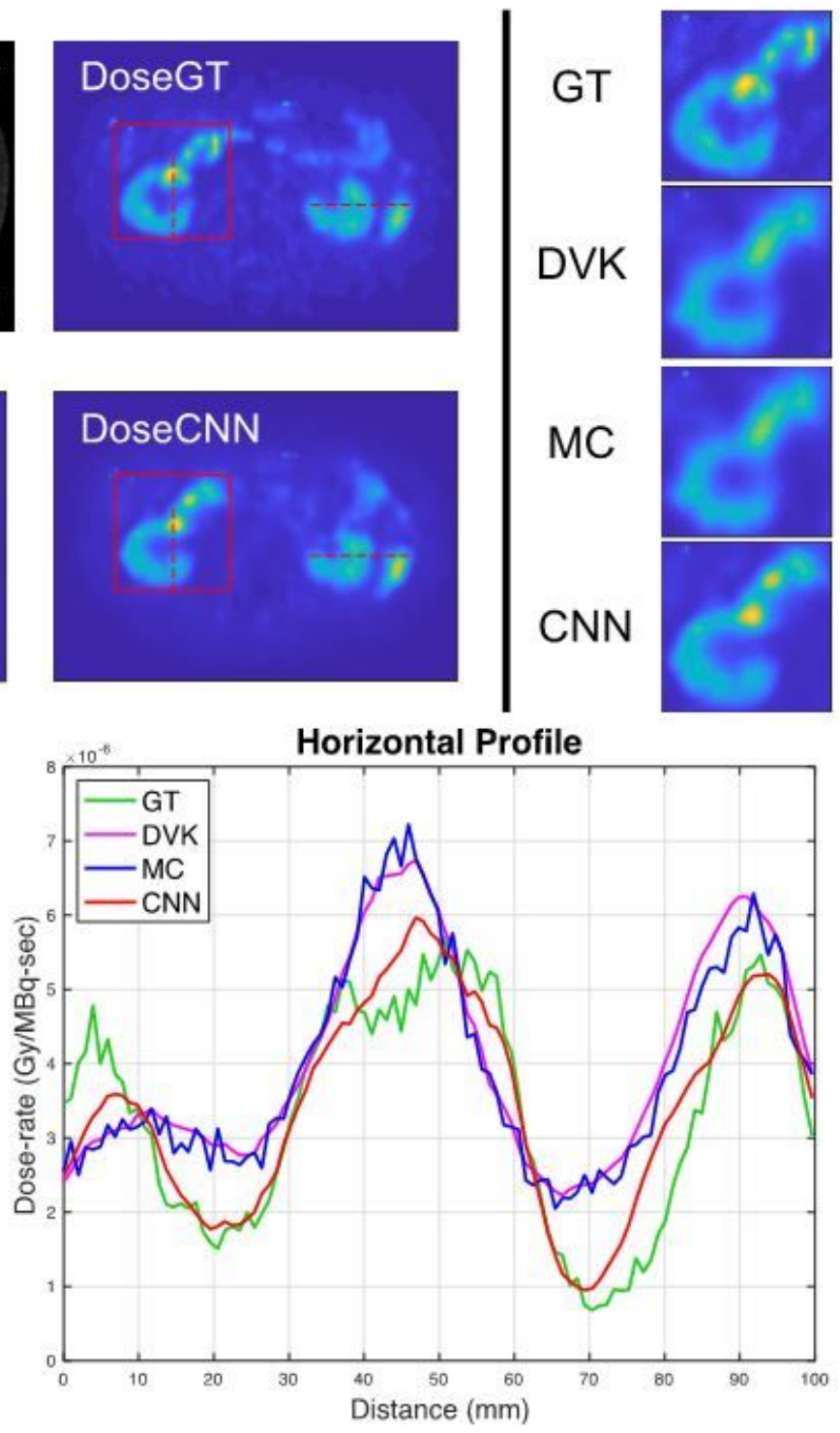

Figure 3

One slice of the input images (SPECT, CT), the DVK, MC, CNN and ground-truth dose-rate map and line profiles corresponding to test phantom \#2. 
A.
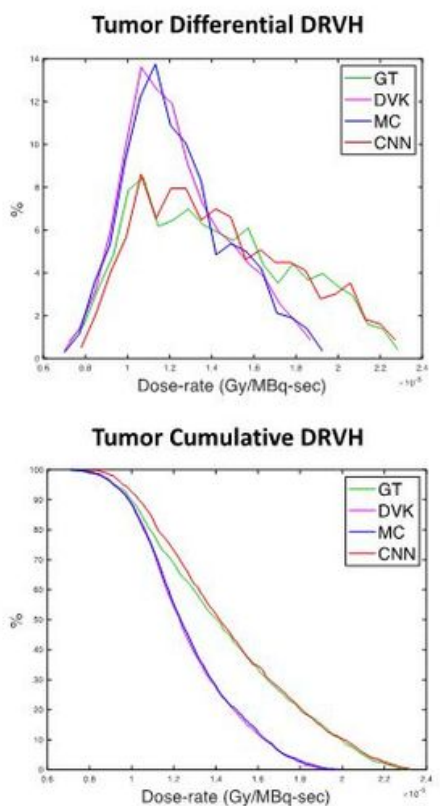

Kidney Differential DRVH

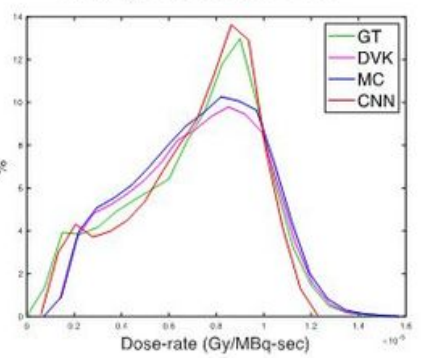

Kidney Cumulative DRVH

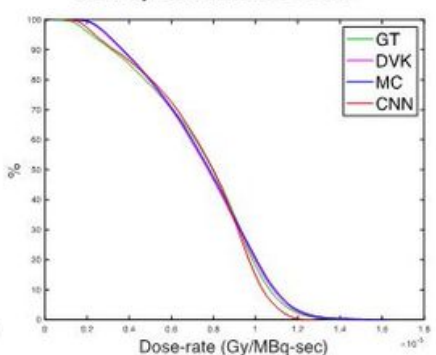

B.
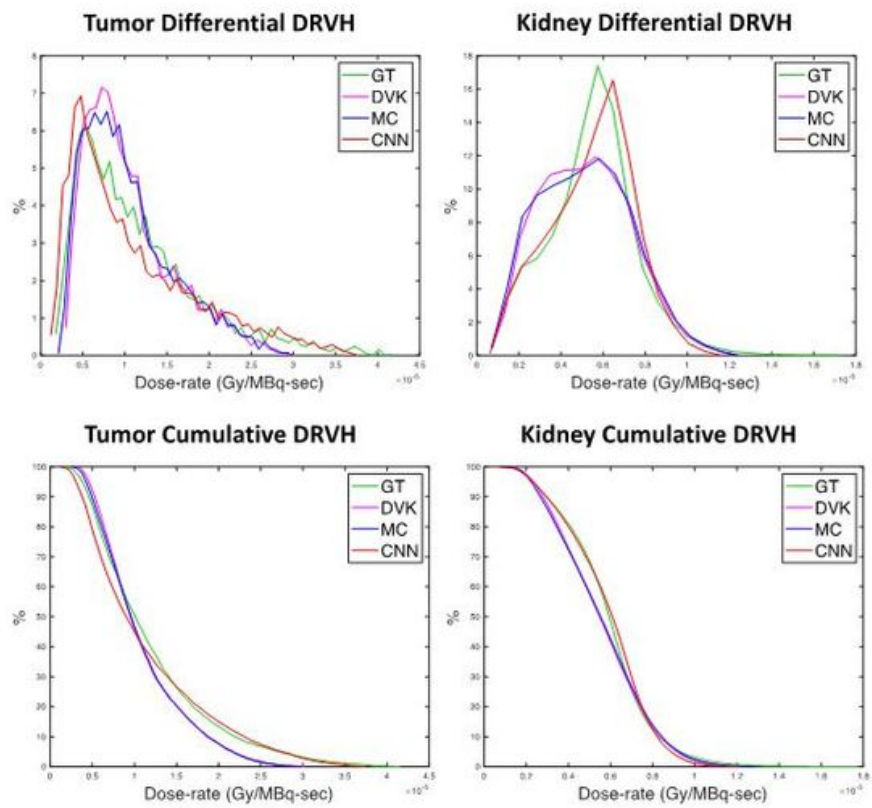

\section{Figure 4}

Differential and cumulative dose-rate volume histograms corresponding to DVK, MC, CNN and the ground-truth dose-rate maps. (A) a $4 \mathrm{~mL}$ tumor and the kidney for phantom \#1; (B) a $10 \mathrm{~mL}$ tumor and the kidney for phantom \#5.
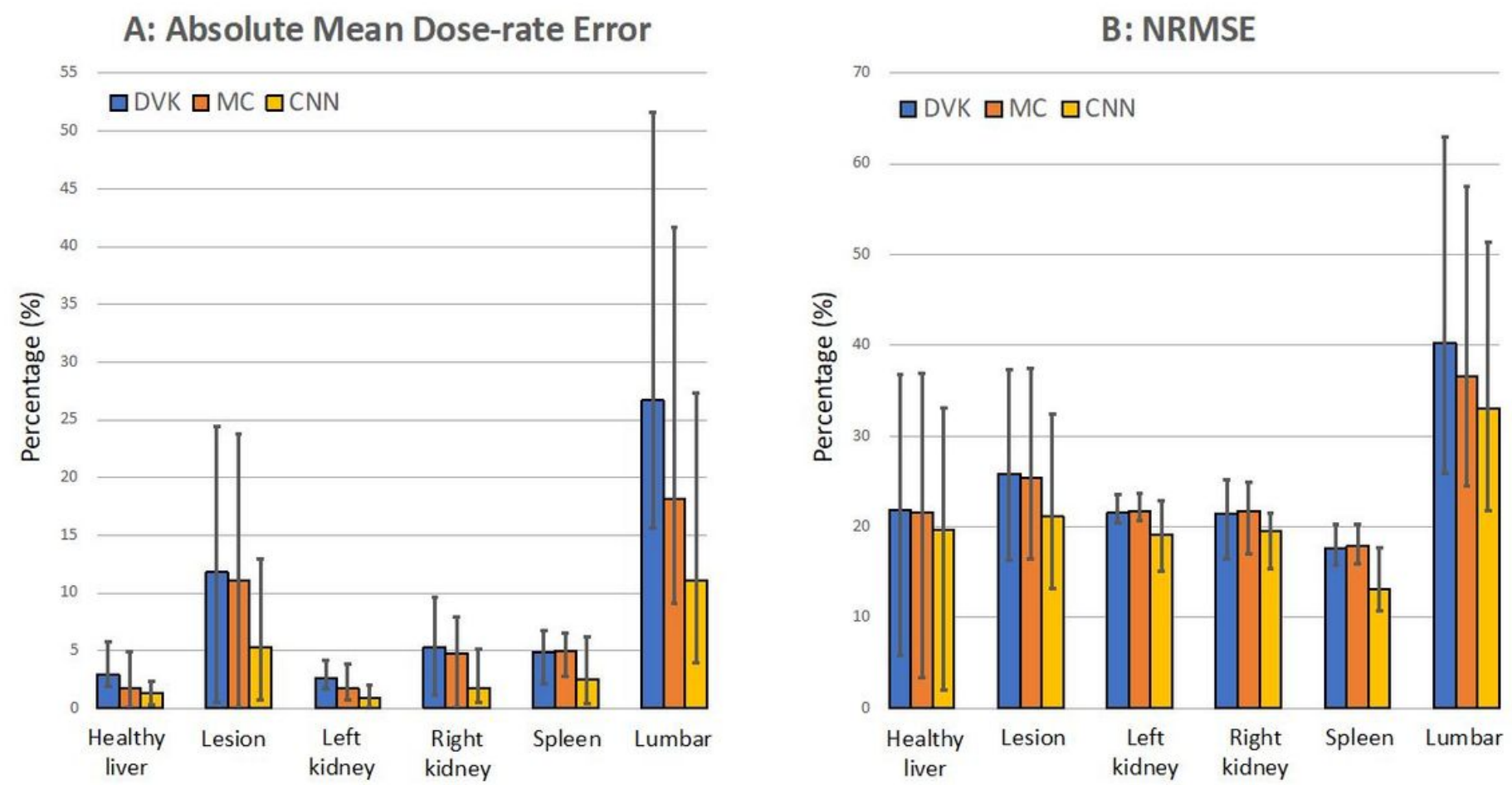

Figure 5 
Comparison of DVK, MC and CNN performance relative to ground-truth dose-rate map across all test phantoms. (A): Absolute mean dose-rate error; (B): NRMSE. The lesion volumes ranged from 4-181 mL.

A.
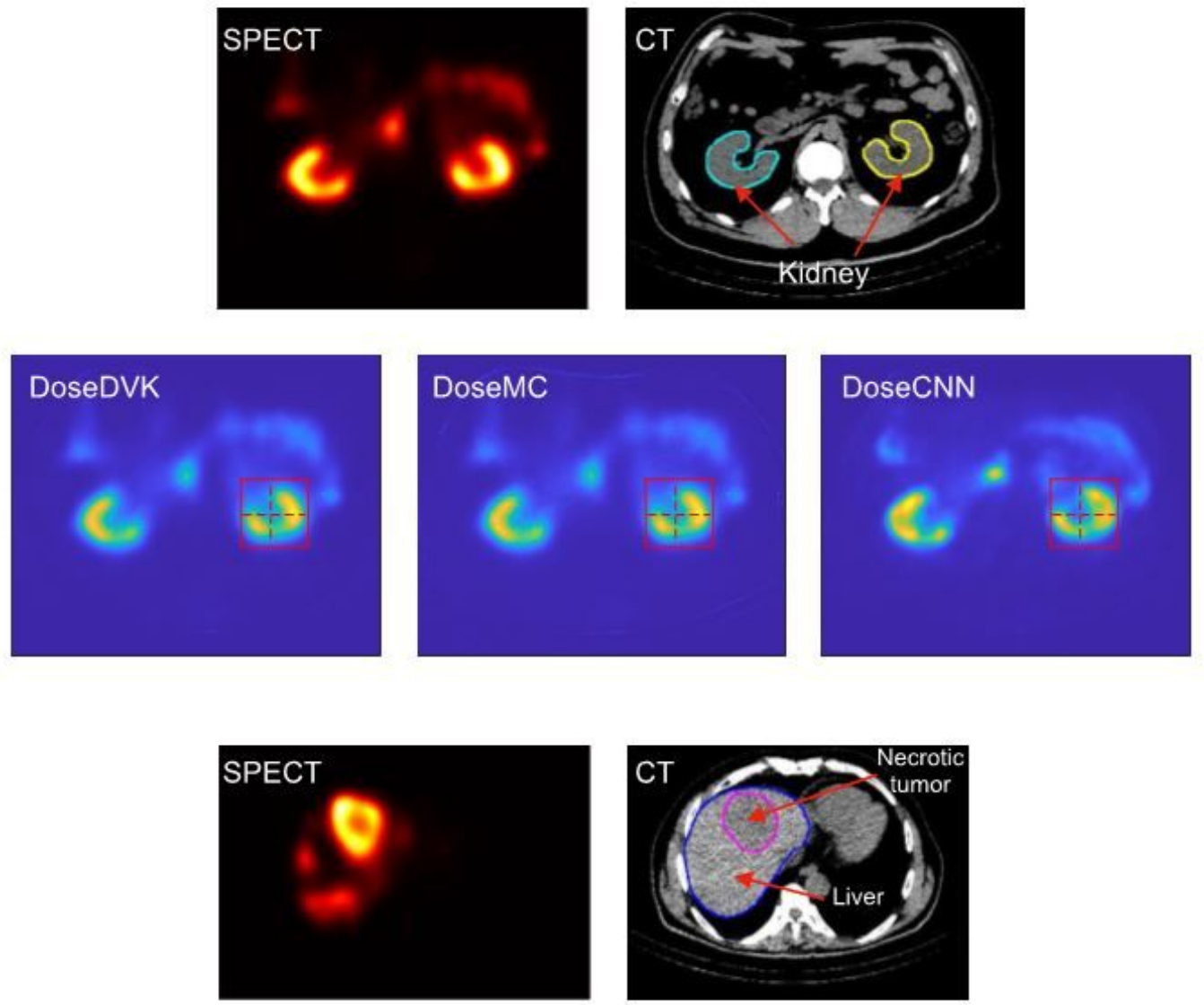

B.
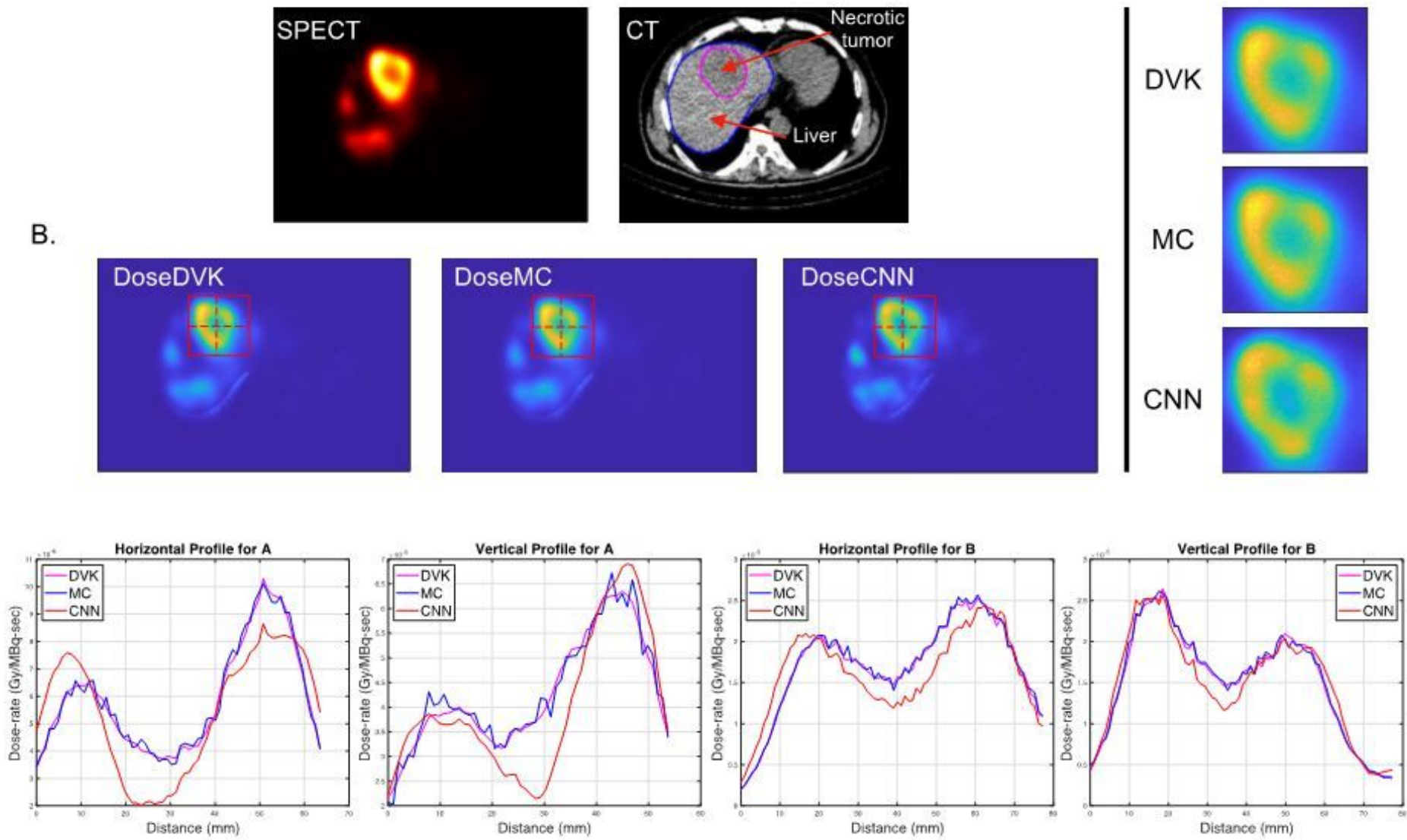

Figure 6

One slice of the input images (SPECT, CT) and DVK, MC, CNN dose-rate maps and line profiles for two patients imaged after Lu-177 DOTATATE. (A) at day 1 post-therapy; (B) at day 7 post-therapy. 


\section{CNN vs. MC}

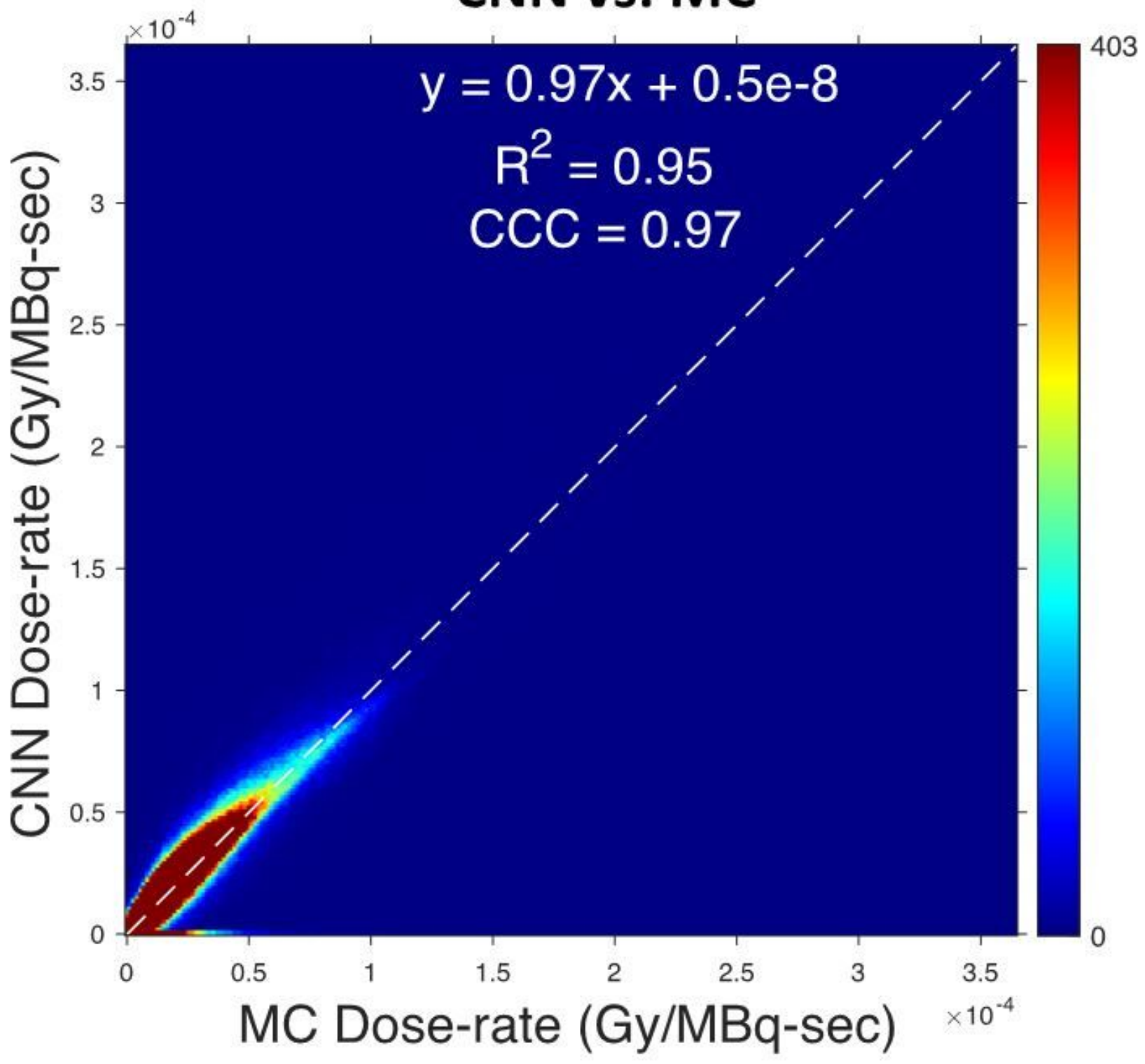

Figure 7

Joint histogram analysis of CNN vs. MC dose-rate across all VOIs in all test patients.

\section{Supplementary Files}

This is a list of supplementary files associated with this preprint. Click to download.

- supplementdose20210305.docx 